\title{
Modeling the relationship between body weight and energy intake: A molecular diffusion-based approach
}

Zhejun Gong $^{1 *}$ and Zhefeng Gong ${ }^{2^{*}}$

\begin{abstract}
Background: Body weight is at least partly controlled by the choices made by a human in response to external stimuli. Changes in body weight are mainly caused by energy intake. By analyzing the mechanisms involved in food intake, we considered that molecular diffusion plays an important role in body weight changes. We propose a model based on Fick's second law of diffusion to simulate the relationship between energy intake and body weight.

Results: This model was applied to food intake and body weight data recorded in humans; the model showed a good fit to the experimental data. This model was also effective in predicting future body weight.

Conclusions: In conclusion, this model based on molecular diffusion provides a new insight into the body weight mechanisms.

Reviewers: This article was reviewed by Dr. Cabral Balreira (nominated by Dr. Peter Olofsson), Prof. Yang Kuang and Dr. Chao Chen.
\end{abstract}

Keywords: Molecular diffusion, Body weight, Model, Choice making

\section{Background}

Body weight change is a complex behavioral response associated with appetite regulation and energy metabolism [1]. Although changes in body weight involve genetic, metabolic, biochemical, cultural and psychosocial factors, the two main factors that regulate body weight are food intake and energy expenditure [2,3]. In recent years, mathematical models have become increasingly used in medical research. These models have helped researchers to develop new ways of dealing with animal behaviors. In terms of body weight, behavioral economic models have been developed to address the effects of environmental factors on energy intake and body weight [4]. A series of experimental studies have also been conducted to develop mathematical models to describe the physiological basis of body weight. In fact, these models can quantitatively address the

\footnotetext{
* Correspondence: zhejungong@yahoo.com.cn; zfgong@gmail.com ${ }^{1}$ College of Logistics Engineering, Wuhan University of Technology, Wuhan, Hubei Province 430063, China

${ }^{2}$ School of Medicine, Zhejiang University, Hangzhou, Zhejiang 310058, China
}

metabolic processes underlying body weight changes and can be used to aid body weight control [5-8]. A mathematical model has also been proposed to address the molecular mechanisms underlying body weight, although the validity of the model has not been verified experimentally [9].

In this paper, we examined the impacts of energy intake and energy expenditure on body weight. Neuropeptides are small protein-like molecules released by neurons to communicate with each other. These neuronal signaling molecules influence specific activities of the brain, including control of food intake [1,10-12]. Neuropeptides are expressed and released by neurons, and mediate or modulate neuronal communication by acting on cell surface receptors. They have a long halflife, show high affinity for their receptors, and reach their target by diffusion, often over a long distance [13-15]. More specifically, food intake can induce the synthesis of specific neuropeptides that diffuse to activate metabolic processes [10]. Considering the above discussion on the neural regulation of obesity, it seems likely that the

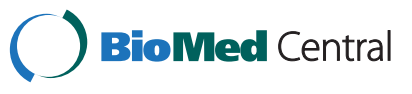


molecular mobility (diffusion) of neuropeptides, for example, plays an important role in body weight regulation. In other words, the body converts food stimuli to molecular signaling processes. The molecular mobility of body weight control is at least partly explained by the diffusion of molecules inside or outside of neural cells. Accordingly, changes in body weight are influenced by molecular movements driven by energy intake. Fick's second law, also known as the diffusion equation, describes non-steady-state diffusion, and is typically used to model molecular mobility [16]. Therefore, we can use the molecular diffusion model to describe body weight behavior, replacing molecular concentration with calorie intake as the driving force in this process. It is known that some biological molecules are synthesized at high concentrations and subsequently affect the concentrations of other molecules by diffusion, until the resulting behavior is established. Therefore, we incorporated the diffusion equation as a model of body weight control and validated this model using experimental data. Because the diffusion equation is nonlinear, the correct parameters can be obtained by global optimization.

In summary, we propose a model in which body weight control is derived from molecular diffusion. We also quantitatively investigate the relationship between energy intake and body weight, by applying Fick's second law of diffusion in combination with a mathematical algorithm. Validation of the model with experimental data obtained from humans showed that the model dynamically simulates changes in body weight and energy intake very well. This model is suitable for describing the relationship between energy intake and body weight.

\section{Results and discussion}

Body weight change: a molecular diffusion based process

Because molecular mobility is accompanied by energy transference, we can describe molecular diffusion with energy diffusion. The human body obeys the law of energy conservation [7], which can be expressed as

$$
\frac{d}{d t}(\rho * V)=\frac{d E}{d t}
$$

where $\rho$ is the energy density of body mass, $V$ is the body mass, $E$ is the net energy intake, $t$ is the time.

Suppose $J$ is energy flux (amount of energy per unit area per unit time in direction $x$ ), $\mathrm{p}$ is the energy density of body fat mass. For healthy adults (18-59y), body weight changes largely due to fat mass (FM) [17], so $d$ $(\rho * \mathrm{~V})$ is approximately equal to $\mathrm{p} * \mathrm{dV}$. We have

$$
\frac{d E}{d t}=-\frac{d J}{d x}
$$

and

$$
J=-D \frac{p d V}{d x}
$$

where $D$ is energy diffusion coefficient. Substituting Equation 2 and Equation 3 into Equation 1 leads to the following equation:

$$
\frac{d V}{d t}=D \frac{d^{2} V}{d x^{2}}
$$

Equation 4 is actually the form of Fick's second law of diffusion.

In the initial conditions where $t=0$ and $x>0$, then $V=V_{0}$. In marginal conditions where $t>0$ and $x=0$, then $V=V_{s}$. When $t>0$ and $x=\infty, V=V_{O} . V_{O}$ is the initial body mass, $V_{s}$ is the body mass transformed from energy intake. Therefore, the solution of Equation 4 is:

$$
\begin{aligned}
V(x, t) & =V_{s}[1-\operatorname{erf}(x /(2 \sqrt{D t}))] \\
& +V_{0} \operatorname{erf}(x /(2 \sqrt{D t}))
\end{aligned}
$$

where $\operatorname{erf}(c)=(2 / \sqrt{\pi}) \int_{0}^{c} \exp \left(-c^{2}\right) d c$. Because $V_{s}=E / p$, Equation 5 can be rewritten as the following equation:

$$
\begin{aligned}
V(t) & =\frac{1}{p} E[1-\operatorname{erf}(x /(2 \sqrt{D t}))] \\
& +V_{0} \operatorname{erf}(x /(2 \sqrt{D t}))
\end{aligned}
$$

From the above discussion, we can know the body weight change process is a diffusion process driven by energy intake.

\section{Fitting and the model to experimental data and validation}

As described above, changes in body weight can be explained by molecular movement driven by energy intake. Considering that body weight change mimics molecular diffusion, and that diffusive processes are involved in body weight changes at the cellular level, this behavioral activity can be described by Equation 6 .

To use the molecular diffusion based model to describe the relationship between energy intake and body weight, because distance $x$ represents body attributes, it is set as a constant in this model. In this way, Equation 6 can be rewritten as:

$$
f(t)=b^{*} \operatorname{erf}(\beta / \sqrt{t})+\alpha^{*} l^{*}[1-\operatorname{erf}(\beta / \sqrt{t})]
$$

where $f(t)=$ body weight, $b=$ initial body weight, $l=$ energy intake, $t=$ time of feeding, and $\alpha$ and $\beta$ are 
constants. If $t>1$, this formula can be rewritten as follows:

$$
f(t)=f(t-1)^{*} \operatorname{erf}(\beta)+\alpha^{*} l(t)^{*}[1-\operatorname{erf}(\beta)]
$$

where $l(t)=$ energy intake, with other parameters identical to those in Equation 7.

Equation 8 can then be applied to simulate experimental data and its validity tested against reference data (in this case human body weight). To best estimate the model parameters, ISCEM algorithm was adopted because this algorithm can not only estimate parameters in complex functions but also conduct global optimization [18]. Energy intake and body weight were recorded for humans in an earlier study [19]. If the experimental data and model-derived data show a good fit, we can conclude that the model is suitable to describe the relationship between energy intake and body weight.

\section{Simulation of body weight change using the developed model}

Using the experimental data recorded over 24 weeks (Table 1) and the ISCEM algorithm, the following constants were obtained:

$\alpha=0.016337, \beta=1.7096$

Entering these constants yields the following equation:

$$
\begin{aligned}
f(t) & =f(t-1)^{*} \operatorname{erf}(1.7096)+0.016337^{*} 7 \\
& * l(t)^{*}[1-\operatorname{erf}(1.7096)]
\end{aligned}
$$

Using Equation 9, we can estimate daily body weight from week S1 to week S24. The model-generated body weight data are plotted alongside the actual experimental data in Figure 1. The determination coefficient $\left(R^{2}\right)$ for this plot was 0.99666 , which indicates that the modelgenerated data closely match the experimental data. Comparison between the actual experimental body weight and model result of each subject is shown in Appendix A.

\section{Model validation and body weight prediction}

We next sought to validate the model. To achieve this, body weight measured between week S1 and week S12 from Table 1 were entered into the ISCEM algorithm, which yielded the following constants:

$$
\alpha=0.0170757, \beta=1.7029
$$

Entering these constants into Equation 8 yields the following equation:

$$
\begin{aligned}
f(t) & =f(t-1)^{*} \operatorname{erf}(1.7029)+0.0170757^{*} 7 \\
& * l(t)^{*}[1-\operatorname{erf}(1.7029)]
\end{aligned}
$$

\begin{tabular}{|c|c|c|c|c|}
\hline $\begin{array}{l}\text { Time } \\
\text { (week) }\end{array}$ & $\begin{array}{l}\text { Body } \\
\text { weight } \\
\text { (kg) }\end{array}$ & $\begin{array}{l}\text { TEE } \\
\text { (kcal/day) }\end{array}$ & $\begin{array}{l}\text { Mean energy } \\
\text { intake } \\
\text { (kcal/day) }\end{array}$ & $\begin{array}{l}\text { Net energy } \\
\text { intake } \\
\text { (kcal/day) }\end{array}$ \\
\hline 0 & 69.39 & 1934.33 & 3538.72 & 1604.39 \\
\hline S1 & 68.35 & 1884.53 & 1658 & -226.53 \\
\hline S2 & 66.8 & 1835.21 & 1658 & -177.21 \\
\hline S3 & 65.76 & 1786.36 & 1648.88 & -137.48 \\
\hline S4 & 64.29 & 1737.6 & 1610.88 & -126.72 \\
\hline S5 & 63.33 & 1691.55 & 1645.94 & -45.61 \\
\hline S6 & 62.16 & 1643.45 & 1639.16 & -4.29 \\
\hline S7 & 61.11 & 1595.75 & 1639.03 & 43.28 \\
\hline S8 & 60.31 & 1548.28 & 1634.84 & 86.56 \\
\hline S9 & 59.56 & 1500.8 & 1620.41 & 119.61 \\
\hline S10 & 58.71 & 1453.31 & 1595.31 & 142 \\
\hline S11 & 58.14 & 1405.63 & 1578.72 & 173.09 \\
\hline S12 & 57.28 & 1357.94 & 1525.16 & 167.22 \\
\hline S13 & 56.6 & 1346.8 & 1515.69 & 168.89 \\
\hline S14 & 56.16 & 1335.67 & 1492.84 & 157.17 \\
\hline S15 & 55.69 & 1324.54 & 1459.94 & 135.4 \\
\hline S16 & 54.7 & 1313.39 & 1430.5 & 117.11 \\
\hline S17 & 54.28 & 1302.28 & 1488.81 & 186.53 \\
\hline S18 & 54.08 & 1291.1 & 1486.44 & 195.34 \\
\hline S19 & 53.51 & 1281.22 & 1519.72 & 238.5 \\
\hline S20 & 53.18 & 1271.34 & 1515.47 & 244.13 \\
\hline S21 & 52.99 & 1261.46 & 1538.75 & 277.29 \\
\hline S22 & 52.9 & 1251.58 & 1554.06 & 302.48 \\
\hline S23 & 52.83 & 1241.7 & 1581.19 & 339.49 \\
\hline S24 & 52.57 & 1231.83 & 1641.63 & 409.8 \\
\hline
\end{tabular}

Using Equation 10, it is possible to estimate the daily body weight from week S1 to week S12. The model-
Table 1 Group's body weight related data (S1-S24) from Minnesota human starvation study

generated data are plotted alongside the experimental data in Figure 2. $R^{2}$ for this model was 0.98499 , indicating very close fit between the model and the experimental data.

Finally, we used this model with the parameters based on the experimental data for weeks $\mathrm{S} 1-\mathrm{S} 12$ to predict body weight change between week S13 and week S24. The body weights predicted for weeks S13-S24 and the corresponding experimental data are plotted in Figure 3. The $\mathrm{R}^{2}$ for this model was 0.94229 , indicating the model satisfactorily fits the experimental data. Confidence intervals for predicted body weight of each subject were provided in Appendix B.

\section{Methods}

\section{Ethics statement}

Because human data were used, approval was obtained from Wuhan University of Technology's Ethics Committee. This research was based on experimental data from 


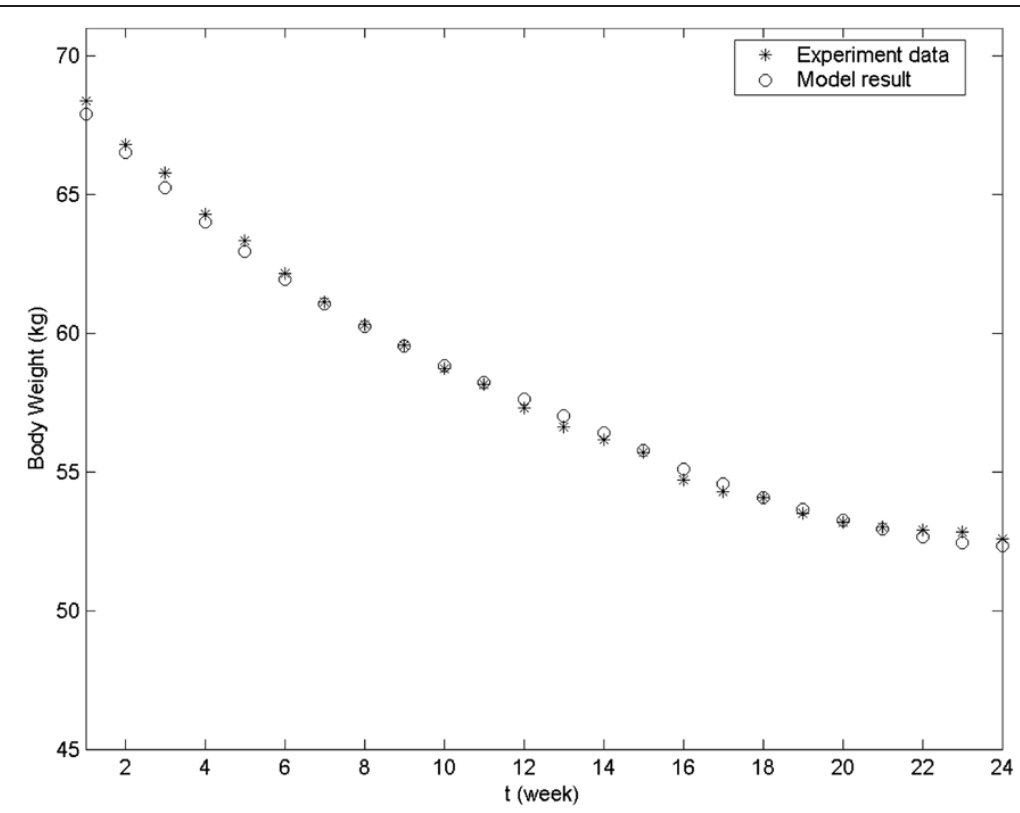

Figure 1 Comparison of experimentally recorded and model-generated group's body weight of humans (weeks S1-S24). Asterisks, experimentally recorded data; circles, model-generated data.

literature [19]. As such, no consent statement for participation is required.

\section{The Minnesota starvation study}

The study reduced the energy intake of 32 male conscientious objectors (20-33 y old, mean 25.5 y) to decrease body mass comparably to severely undernourished prisoners of war with the aim of testing methods for rehabilitating starved men. The study included a 12-week control phase (weeks C1-C12), 24 weeks of energy restriction (weeks S1-S24), and 20 weeks of recovery (R1-R20). During weeks $\mathrm{C} 1-\mathrm{C} 12$, energy intake was adjusted to bring individuals towards the group norm, based on weight for height, with a mean weight loss of $0.80 \mathrm{~kg}$. Physical activity included 22 miles per week of outdoor walking and additional walking on campus, plus custodial

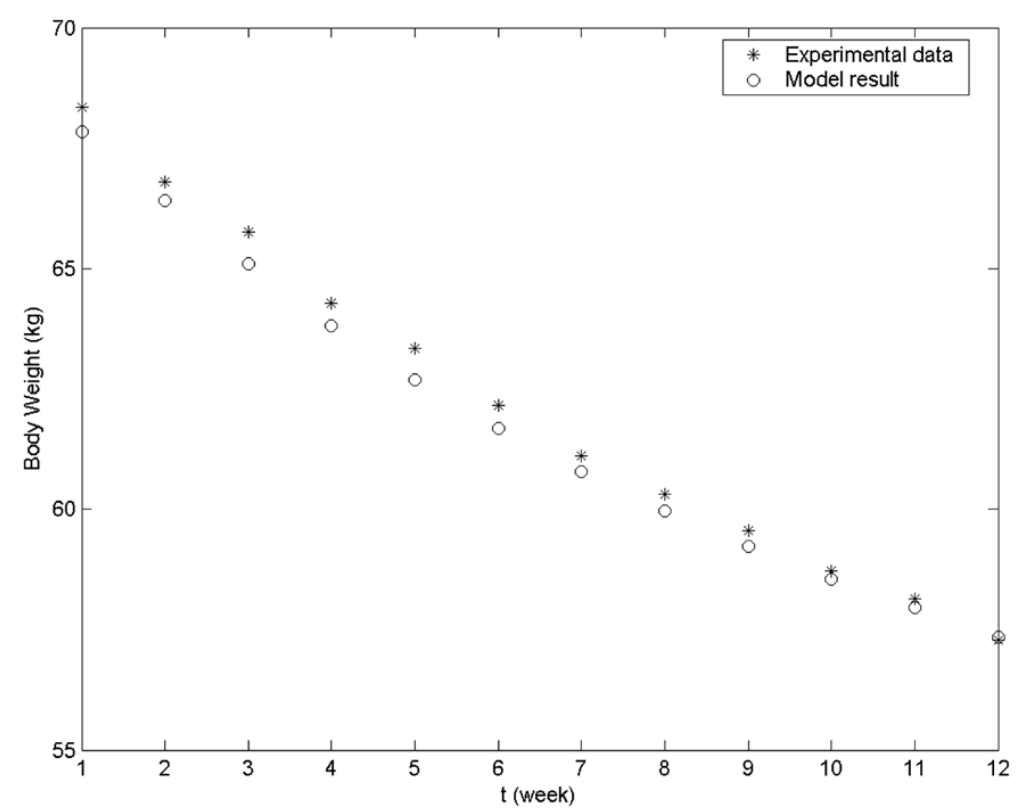

Figure 2 Comparison of experimentally recorded and model-generated group's body weight of humans (weeks S1-S12). Asterisks, experimentally recorded data; circles, model-generated data. 


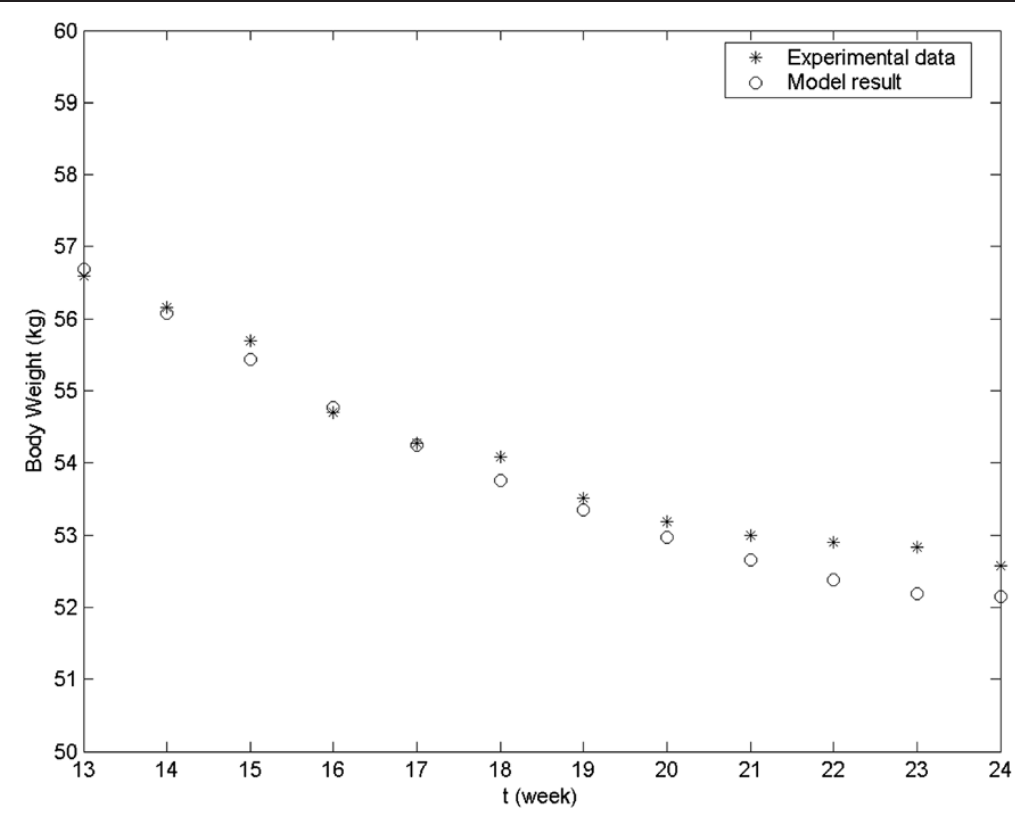

Figure 3 Prediction of group's body weight during weeks S13-S24 based on actual experimental data from weeks S1-S12. The actual experimental data in weeks S13-S24 are also shown. Asterisks, actual experimental data; circles, model-generated data.

duties. All subjects were also required to walk at 3.5 miles per hr for half an hr per week on a motor-driven treadmill with a $10 \%$ grade. The control diet contained about $100 \mathrm{~g}$ of protein, $400 \mathrm{~g}$ of carbohydrates, and $130 \mathrm{~g}$ of fat. Energy intake averaged $3,492 \mathrm{kcal} / \mathrm{d}(14.62 \mathrm{~mJ} / \mathrm{d})$ for the last 3 control weeks, during which group weight declined only $0.3 \mathrm{~kg}$. From then on, subjects were fed at a level that was expected to cause a $24 \%$ group average decrease in body mass during the next 24 weeks. Weight loss was induced by reducing food intake to two daily meals with $51 \mathrm{~g}$ of protein, $286 \mathrm{~g}$ of carbohydrates, and $30 \mathrm{~g}$ of fat, with 3 basic menus consisting of cereal, whole-wheat bread, potatoes, turnips, and cabbage, supplemented by scant amounts of meat and dairy products. During the entire starvation period, walking 22 miles a week and custodial work remained mandatory [19].

Total Energy Expenditure (TEE) includes two major parts: Resting Energy Expenditure (REE), the amount of calories needed to maintain basic body systems and body temperature at rest; Activity Energy Expenditure (AEE), the amount of calories used during activity [20]. Net energy intake is the difference between food intake and TEE. Although TEE was not measured in the Minnesota starvation study, TEE can be obtained through calculating REE and AEE [19,21].

Some useful data are shown in Table 1.

\section{ISCEM algorithm: an improved SCEM-UA algorithm}

The shuffled complex evolution metropolis algorithm (SCEM-UA) is a global-searching algorithm developed by Vrugt JA et al. [22]. The SCEM-UA method adopts Markov Chain Monte Carlo theory (MCMC) and uses the Metropolis-Hastings algorithm (MH), replacing the Downhill Simplex method, to obtain a global optimal estimation. Although SCEM-UA can successfully obtain the global optimal solution, its performance depends on correct setting of the minimal and maximal limits. In the current study, we improve the SCEM-UA algorithm so that it can optimize the parameter searching space and obtain the optimal solution. This improved algorithm is termed the ISCEM algorithm.

Suppose $\hat{y}=\eta(\xi \mid \theta)$, where $\hat{y}$ is an $N \times 1$ vector of model predictions, $\xi$ is an $N \times n$ matrix of input variables and $\theta$ is a vector of $n$ unknown parameters. The SCEM-UA algorithm is given below:

(1) To initialize the process, choose the population size $s$ and the number of complexes $q$. The algorithm tentatively assumes that the number of sequences is identical to the number of complexes.

(2) Generate $s$ samples from the prior distribution $\left\{\theta_{1}, \theta_{2}, \ldots, \theta_{s}\right\}$ and compute the posterior density $\left\{p\left(\theta^{(1)} \mid \mathbf{y}\right), \boldsymbol{p}\left(\theta^{(2)} \mid \mathbf{y}\right), \ldots, p\left(\theta^{(\mathbf{s})} \mid \mathbf{y}\right)\right\}$ at each point [22].

(3) Sort the points in order of decreasing posterior density and store them in an array $\mathrm{D}[1: s, 1: n+1]$, where $n$ is the number of parameters, so that the first row of $\mathrm{D}$ represents the point with the highest posterior density. The extra column stores the posterior density. Initialize the 
starting points of the parallel sequences, $S^{1}, S^{2}, \ldots, S^{q}$, such that $S^{k}$ is $\mathrm{D}[k, 1: \mathrm{n}+1]$, where $k=1,2, \ldots, q$.

(4) Partition $\mathrm{D}$ into $q$ complexes $\mathrm{C}^{\mathrm{l}}, \mathrm{C}^{2}, \ldots, \mathrm{C}^{q}$, each containing $m$ points, such that the first complex contains every $q(j-1)+1$ ranked point, the second complex contains every $q(j-1)+2$ ranked point of $\mathrm{D}$, and so on, where $j=1,2, \ldots, m$.

(5) Initialize $L, T, A R_{\min }, c_{n}$. For each $C^{k}$, call the SEM algorithm [22] and run it L times;

(6) Unpack all complexes $C$ back into D and rank the points in order of decreasing posterior density.

(7) Check Gelman and Rubin (GR) convergence statistic. If convergence criteria are satisfied, stop; otherwise, return to step 4.

The ISCEM algorithm is given below:

1) Suppose $I_{\min } \leq \theta \leq I_{\max }, I_{\min }$ and $I_{\max }$ are interval vectors of $\theta$. The initial $I_{\max }$ is set to be very large. Run the SCEM-UA algorithm and let the output parameter vector with highest posterior density $\left(p_{o}\right)$ be $\theta_{o}$. Set $\mathrm{I}_{\max }=\theta_{o}$.

2) Run the SCEM-UA algorithm again, and let the output parameter vector with highest posterior density $\left(p_{w}\right)$ be $\theta_{w}$. If $\left\|p_{o}-p_{w}\right\| \leq \epsilon$, where $\epsilon>0$, go to step (4); otherwise set $\theta_{o}=\theta_{w}$.

3) If $p_{o} \leq p_{w}$, let $\mathrm{I}_{\max }=\theta_{w}$; otherwise, let $\mathrm{I}_{\min }=\theta_{w}$. Let $p_{o}=p_{w}$, go to step (2).

4) Output $\theta_{w}$.

\section{Conclusions}

In this paper, we have shown that energy intake and energy expenditure in humans can be simulated using a mathematical algorithm based on molecular diffusion. In the model, only the effects of calorie intake on body weight are considered; other variables that may affect body weight are included as constants. This is because the internal and external environmental factors that may influence body weight can be assumed to be stable when environment is stable. In fact, as shown here, if these factors are kept relatively stable, the prediction of body weight based on energy intake and defined constants matches closely with experimental data.

In this model, only the general relationship between energy intake and body weight was examined. We believe this model will provide new insights into the mechanisms underlying body weight control. In future studies, more information is needed to examine the impact of neuronal signaling mechanisms that control body weight on this model.

\section{Appendix}

Appendix A Table 2

Table 2 Comparison of experimental data and Model result of each subject

\begin{tabular}{|c|c|c|c|c|c|c|c|c|c|c|c|c|}
\hline No. & $\begin{array}{l}\text { Weight } \\
\text { S1 } \\
\text { kg }\end{array}$ & $\begin{array}{l}\text { Weight } \\
\text { S2 } \\
\text { kg }\end{array}$ & $\begin{array}{l}\text { Weight } \\
\text { S3 } \\
\text { kg }\end{array}$ & $\begin{array}{l}\text { Weight } \\
\text { S4 } \\
\text { kg }\end{array}$ & $\begin{array}{l}\text { Weight } \\
\text { S5 } \\
\text { kg }\end{array}$ & $\begin{array}{l}\text { Weight } \\
\text { S6 } \\
\text { kg }\end{array}$ & $\begin{array}{l}\text { Weight } \\
\text { S7 } \\
\text { kg }\end{array}$ & $\begin{array}{l}\text { Weight } \\
\text { S8 } \\
\text { kg }\end{array}$ & $\begin{array}{l}\text { Weight } \\
\text { S9 } \\
\text { kg }\end{array}$ & $\begin{array}{l}\text { Weight } \\
\text { S10 } \\
\text { kg }\end{array}$ & $\begin{array}{l}\text { Weight } \\
\text { S11 } \\
\text { kg }\end{array}$ & $\begin{array}{l}\text { Weight } \\
\text { S12 } \\
\text { kg }\end{array}$ \\
\hline $122(p)$ & 64.6 & 63 & 61.6 & 60.2 & 59 & 57.6 & 56.6 & 55.5 & 54.6 & 53.5 & 52.8 & 52.1 \\
\hline $122(\mathrm{~m})$ & 63.974 & 62.658 & 61.434 & 60.249 & 59.226 & 58.294 & 57.461 & 56.718 & 56.046 & 55.424 & 54.867 & 54.309 \\
\hline 123(p) & 63.8 & 62.6 & 61.8 & 60.6 & 60.1 & 59.1 & 58.2 & 57.6 & 57.3 & 56.8 & 56.5 & 55.8 \\
\hline $123(\mathrm{~m})$ & 63.285 & 61.98 & 60.767 & 59.591 & 58.579 & 57.657 & 56.834 & 56.101 & 55.438 & 54.826 & 54.279 & 53.73 \\
\hline $119(p)$ & 65.5 & 64.1 & 63 & 61.5 & 60.7 & 59.4 & 58.4 & 57.6 & 56.9 & 55.9 & 55.4 & 54.5 \\
\hline $119(\mathrm{~m})$ & 64.86 & 63.531 & 62.293 & 61.094 & 60.058 & 59.113 & 58.267 & 57.511 & 56.827 & 56.193 & 55.624 & 55.054 \\
\hline $120(p)$ & 69.6 & 68.2 & 67.1 & 65.6 & 64.4 & 63.2 & 61.9 & 61.1 & 60.5 & 59.7 & 58.7 & 57.7 \\
\hline $120(\mathrm{~m})$ & 69.29 & 67.891 & 66.585 & 65.319 & 64.218 & 63.207 & 62.297 & 61.479 & 60.732 & 60.037 & 59.409 & 58.78 \\
\hline 129(p) & 64.7 & 63.3 & 62.4 & 61 & 60.3 & 59.6 & 58.6 & 58.1 & 57.7 & 57 & 57 & 56.1 \\
\hline $129(\mathrm{~m})$ & 64.171 & 62.852 & 61.625 & 60.436 & 59.411 & 58.476 & 57.64 & 56.894 & 56.219 & 55.595 & 55.036 & 54.475 \\
\hline 130(p) & 64.8 & 63.4 & 63 & 61.5 & 60.7 & 60.1 & 59 & 58.5 & 58 & 58.1 & 57.8 & 57.5 \\
\hline 130(m) & 64.565 & 63.24 & 62.007 & 60.812 & 59.781 & 58.84 & 57.998 & 57.247 & 56.566 & 55.937 & 55.372 & 54.806 \\
\hline 126(p) & 82.6 & 81.2 & 79.8 & 78.1 & 77.1 & 75.4 & 74.1 & 72.8 & 71.8 & 70.6 & 69.8 & 69.1 \\
\hline $126(\mathrm{~m})$ & 81.89 & 80.294 & 78.795 & 77.338 & 76.049 & 74.853 & 73.762 & 72.764 & 71.842 & 70.973 & 70.174 & 69.377 \\
\hline $127(p)$ & 63.1 & 61.2 & 60.2 & 58.3 & 57.4 & 56.1 & 55.5 & 54.6 & 54.2 & 53.2 & 52.9 & 52.5 \\
\hline $127(\mathrm{~m})$ & 62.793 & 61.496 & 60.29 & 59.122 & 58.117 & 57.202 & 56.386 & 55.66 & 55.004 & 54.399 & 53.858 & 53.316 \\
\hline $22(p)$ & 64.2 & 62.8 & 61.4 & 60.2 & 59.2 & 58.1 & 57.2 & 56.8 & 56.2 & 55.4 & 55 & 53.8 \\
\hline
\end{tabular}


Table 2 Comparison of experimental data and Model result of each subject (Continued)

\begin{tabular}{|c|c|c|c|c|c|c|c|c|c|c|c|c|}
\hline $22(m)$ & 63.679 & 62.368 & 61.148 & 59.967 & 58.949 & 58.021 & 57.192 & 56.453 & 55.785 & 55.168 & 54.615 & 54.061 \\
\hline $23(p)$ & 68.3 & 66.6 & 65.4 & 64 & 62.8 & 61.6 & 60.4 & 59.6 & 58.5 & 57.6 & 56.9 & 55.8 \\
\hline $23(m)$ & 67.715 & 66.341 & 65.059 & 63.817 & 62.739 & 61.751 & 60.864 & 60.068 & 59.344 & 58.67 & 58.063 & 57.455 \\
\hline 19(p) & 69.6 & 68.3 & 67.6 & 65.9 & 64.6 & 63.7 & 62.6 & 61.7 & 60.8 & 59.4 & 58.6 & 57.5 \\
\hline $19(m)$ & 69.093 & 67.697 & 66.395 & 65.131 & 64.033 & 63.025 & 62.118 & 61.302 & 60.559 & 59.867 & 59.241 & 58.614 \\
\hline $20(p)$ & 63.7 & 62.5 & 61.5 & 60.1 & 59 & 58 & 57.1 & 56.4 & 55.5 & 54.9 & 54.7 & 53.9 \\
\hline $20(m)$ & 63.285 & 61.98 & 60.767 & 59.591 & 58.579 & 57.657 & 56.834 & 56.101 & 55.438 & 54.826 & 54.279 & 53.73 \\
\hline 29(p) & 69.7 & 68.1 & 67.3 & 66.5 & 65.5 & 65.4 & 64.5 & 63 & 60.3 & 57.4 & 55.1 & 54.5 \\
\hline $29(\mathrm{~m})$ & 69.585 & 68.182 & 66.871 & 65.601 & 64.495 & 63.48 & 62.566 & 61.743 & 60.993 & 60.294 & 59.661 & 59.028 \\
\hline 30(p) & 67.1 & 65.6 & 64.6 & 63.1 & 62.3 & 61 & 60.4 & 59.3 & 59 & 58.2 & 58.6 & 57.6 \\
\hline 30(m) & 66.632 & 65.275 & 64.01 & 62.784 & 61.722 & 60.75 & 59.879 & 59.098 & 58.389 & 57.731 & 57.138 & 56.545 \\
\hline $26(p)$ & 70.3 & 69.3 & 67.7 & 65.8 & 65 & 63.5 & 62.3 & 61.7 & 60.6 & 59.7 & 59.3 & 58.1 \\
\hline $26(\mathrm{~m})$ & 69.782 & 68.376 & 67.062 & 65.789 & 64.68 & 63.662 & 62.745 & 61.92 & 61.166 & 60.465 & 59.829 & 59.194 \\
\hline $27(p)$ & 74.5 & 73.2 & 72 & 69.6 & 68.4 & 67 & 65.5 & 64.4 & 63.1 & 61.9 & 61.5 & 60.8 \\
\hline $27(m)$ & 73.621 & 72.155 & 70.782 & 69.451 & 68.285 & 67.21 & 66.238 & 65.358 & 64.551 & 63.797 & 63.109 & 62.423 \\
\hline 4(p) & 60.9 & 59.6 & 58.6 & 57.2 & 56 & 54.9 & 53.8 & 53.1 & 52.3 & 51.5 & 51 & 50.4 \\
\hline $4(m)$ & 60.627 & 59.364 & 58.191 & 57.056 & 56.084 & 55.2 & 54.415 & 53.72 & 53.095 & 52.519 & 52.008 & 51.494 \\
\hline $5(p)$ & 79.6 & 77.9 & 76.4 & 74.8 & 73.4 & 72.2 & 70.6 & 69.6 & 68.2 & 66.9 & 65.5 & 64.6 \\
\hline $5(\mathrm{~m})$ & 79.134 & 77.581 & 76.124 & 74.709 & 73.461 & 72.306 & 71.254 & 70.296 & 69.411 & 68.581 & 67.819 & 67.059 \\
\hline $1(p)$ & 75.5 & 73.8 & 72.1 & 70.1 & 68.8 & 67.3 & 66 & 65.3 & 64.8 & 64.3 & 63.8 & 64.9 \\
\hline $1(\mathrm{~m})$ & 75.59 & 74.093 & 72.69 & 71.329 & 70.133 & 69.03 & 68.029 & 67.122 & 66.287 & 65.505 & 64.791 & 64.078 \\
\hline $2(p)$ & 72.1 & 70.1 & 69.1 & 67.9 & 67 & 65.7 & 64.6 & 63.5 & 62.6 & 61.5 & 61 & 60.1 \\
\hline $2(m)$ & 71.948 & 70.507 & 69.161 & 67.854 & 66.713 & 65.664 & 64.715 & 63.859 & 63.076 & 62.344 & 61.68 & 61.015 \\
\hline $11(p)$ & 65.7 & 63.9 & 62.6 & 61.6 & 60 & 59.1 & 58.2 & 57.6 & 56.7 & 56 & 55.3 & 54.4 \\
\hline $11(\mathrm{~m})$ & 64.958 & 63.627 & 62.388 & 61.188 & 60.151 & 59.204 & 58.356 & 57.599 & 56.914 & 56.278 & 55.709 & 55.137 \\
\hline 12(p) & 79.7 & 77.5 & 75.8 & 74 & 73.2 & 71.5 & 70.4 & 70 & 69.5 & 68.1 & 68.1 & 68.7 \\
\hline $12(m)$ & 79.33 & 77.775 & 76.315 & 74.897 & 73.646 & 72.488 & 71.433 & 70.472 & 69.585 & 68.752 & 67.987 & 67.224 \\
\hline 8(p) & 63.8 & 62.8 & 62.7 & 61.6 & 60.7 & 59.2 & 58.6 & 58.2 & 57.2 & 57.1 & 56.2 & 53.9 \\
\hline $8(m)$ & 63.187 & 61.883 & 60.671 & 59.497 & 58.487 & 57.566 & 56.744 & 56.012 & 55.351 & 54.74 & 54.195 & 53.647 \\
\hline 9(p) & 71.5 & 69.6 & 69.1 & 68 & 67.2 & 66.2 & 64.6 & 64.1 & 63.6 & 64.1 & 63 & 60.4 \\
\hline $9(\mathrm{~m})$ & 71.357 & 69.926 & 68.588 & 67.291 & 66.159 & 65.118 & 64.178 & 63.33 & 62.555 & 61.832 & 61.175 & 60.518 \\
\hline 104(p) & 66.7 & 64.7 & 63.9 & 63 & 62.5 & 61.1 & 60.2 & 59.3 & 58.9 & 58 & 57.6 & 56.6 \\
\hline 104(m) & 66.632 & 65.275 & 64.01 & 62.784 & 61.722 & 60.75 & 59.879 & 59.098 & 58.389 & 57.731 & 57.138 & 56.545 \\
\hline $105(p)$ & 67.4 & 66 & 65.4 & 63.7 & 63 & 61.7 & 61.3 & 59.9 & 59.3 & 58.1 & 57.5 & 56.5 \\
\hline $105(\mathrm{~m})$ & 67.223 & 65.856 & 64.582 & 63.347 & 62.276 & 61.296 & 60.416 & 59.627 & 58.91 & 58.243 & 57.643 & 57.041 \\
\hline $101(p)$ & 63.7 & 62.4 & 61.6 & 60.2 & 59.2 & 58.2 & 57.2 & 56.5 & 55.8 & 55 & 54.5 & 53.6 \\
\hline $101(\mathrm{~m})$ & 63.088 & 61.786 & 60.576 & 59.404 & 58.394 & 57.475 & 56.654 & 55.924 & 55.264 & 54.655 & 54.111 & 53.564 \\
\hline $102(p)$ & 67 & 65.5 & 64.6 & 63.4 & 62.2 & 61 & 59.9 & 59 & 58 & 57.5 & 57.2 & 56.3 \\
\hline $102(\mathrm{~m})$ & 66.435 & 65.081 & 63.819 & 62.596 & 61.537 & 60.568 & 59.7 & 58.922 & 58.215 & 57.56 & 56.97 & 56.379 \\
\hline $111(p)$ & 62.5 & 60.6 & 59.4 & 58.1 & 57.2 & 56 & 54.9 & 54.3 & 53.9 & 53 & 52.9 & 52.1 \\
\hline $111(\mathrm{~m})$ & 61.612 & 60.333 & 59.145 & 57.995 & 57.008 & 56.11 & 55.311 & 54.602 & 53.963 & 53.373 & 52.849 & 52.322 \\
\hline $112(p)$ & 60.6 & 58.9 & 58 & 56.3 & 55.9 & 54.8 & 53.4 & 52.5 & 51.9 & 51.4 & 50.8 & 50.5 \\
\hline $112(\mathrm{~m})$ & 60.332 & 59.073 & 57.905 & 56.774 & 55.806 & 54.927 & 54.147 & 53.456 & 52.834 & 52.263 & 51.756 & 51.246 \\
\hline $108(p)$ & 66 & 64.6 & 63.5 & 62 & 61.4 & 60.6 & 59.8 & 59.8 & 60.5 & 60 & 59.1 & 57.4 \\
\hline 108(m) & 65.451 & 64.112 & 62.865 & 61.657 & 60.613 & 59.658 & 58.804 & 58.04 & 57.347 & 56.705 & 56.129 & 55.551 \\
\hline
\end{tabular}


Table 2 Comparison of experimental data and Model result of each subject (Continued)

\begin{tabular}{|c|c|c|c|c|c|c|c|c|c|c|c|c|}
\hline 109(p) & 78.3 & 76.3 & 75 & 73.4 & 72.5 & 70.9 & 69.6 & 68.6 & 67.8 & 66.9 & 66.3 & 65.4 \\
\hline 109(m) & 77.657 & 76.128 & 74.693 & 73.3 & 72.074 & 70.941 & 69.91 & 68.973 & 68.11 & 67.3 & 66.558 & 65.817 \\
\hline $\begin{array}{l}\text { Weight } \\
\text { S13 } \\
\text { kg }\end{array}$ & $\begin{array}{l}\text { Weight } \\
\text { S14 } \\
\text { kg }\end{array}$ & $\begin{array}{l}\text { Weight } \\
\text { S15 } \\
\text { kg }\end{array}$ & $\begin{array}{l}\text { Weight } \\
\text { S16 } \\
\text { kg }\end{array}$ & $\begin{array}{l}\text { Weight } \\
\text { S17 } \\
\text { kg }\end{array}$ & $\begin{array}{l}\text { Weight } \\
\text { S18 } \\
\text { kg }\end{array}$ & $\begin{array}{l}\text { Weight } \\
\text { S19 } \\
\text { kg }\end{array}$ & $\begin{array}{l}\text { Weight } \\
\text { S20 } \\
\text { kg }\end{array}$ & $\begin{array}{l}\text { Weight } \\
\text { S21 } \\
\text { kg }\end{array}$ & $\begin{array}{l}\text { Weight } \\
\text { S22 } \\
\text { kg }\end{array}$ & $\begin{array}{l}\text { Weight } \\
\text { S23 } \\
\text { kg }\end{array}$ & $\begin{array}{l}\text { Weight } \\
\text { S24 } \\
\text { kg }\end{array}$ & $\mathrm{R}^{2}$ \\
\hline 51.7 & 51.5 & 51 & 50 & 49.4 & 49 & 48.5 & 48.2 & 47.7 & 47.8 & 47.2 & 47.4 & \\
\hline 53.763 & 53.204 & 52.615 & 52.002 & 51.523 & 51.067 & 50.696 & 50.34 & 50.049 & 49.808 & 49.636 & 49.593 & 0.891 \\
\hline 55.2 & 54.9 & 54.8 & 53.9 & 53.4 & 53 & 52 & 51.9 & 51.5 & 52.1 & 52.2 & 52.1 & \\
\hline 53.192 & 52.642 & 52.062 & 51.458 & 50.988 & 50.54 & 50.177 & 49.829 & 49.546 & 49.313 & 49.149 & 49.113 & 0.685 \\
\hline 53.9 & 53.4 & 53.2 & 52.2 & 51.4 & 51 & 50.8 & 50.5 & 50.3 & 50.7 & 49.8 & 49.1 & \\
\hline 54.496 & 53.926 & 53.325 & 52.702 & 52.212 & 51.745 & 51.363 & 50.997 & 50.696 & 50.444 & 50.263 & 50.21 & 0.988 \\
\hline 56.8 & 56 & 55.5 & 54.7 & 54 & 53.4 & 53 & 52.3 & 52.1 & 51.3 & 51.2 & 51.6 & \\
\hline 58.163 & 57.536 & 56.879 & 56.2 & 55.655 & 55.135 & 54.7 & 54.282 & 53.929 & 53.627 & 53.396 & 53.294 & 0.947 \\
\hline 55.8 & 55.5 & 54.8 & 53.8 & 53.4 & 53.4 & 53.3 & 53.2 & 53 & 52.8 & 52.8 & 52.2 & \\
\hline 53.926 & 53.364 & 52.773 & 52.158 & 51.676 & 51.218 & 50.844 & 50.486 & 50.193 & 49.949 & 49.776 & 49.73 & 0.731 \\
\hline 56.9 & 56.6 & 56.6 & 55.4 & 55.7 & 55.7 & 55.7 & 55.6 & 54.2 & 53.9 & 54.5 & 53.6 & \\
\hline 54.252 & 53.685 & 53.089 & 52.469 & 51.982 & 51.519 & 51.141 & 50.778 & 50.48 & 50.232 & 50.054 & 50.004 & 0.104 \\
\hline 68.1 & 67.4 & 67.6 & 66 & 65.7 & 65.4 & 65.3 & 63 & 62 & 62.6 & 61.6 & 60.6 & \\
\hline 68.595 & 67.804 & 66.987 & 66.15 & 65.45 & 64.777 & 64.191 & 63.625 & 63.126 & 62.681 & 62.308 & 62.067 & 0.988 \\
\hline 51.8 & 51.3 & 51.2 & 50.8 & 50.4 & 49.6 & 49.2 & 48.7 & 49.2 & 48.7 & 49.2 & 49.3 & \\
\hline 52.785 & 52.241 & 51.667 & 51.069 & 50.605 & 50.164 & 49.806 & 49.464 & 49.187 & 48.959 & 48.801 & 48.771 & 0.971 \\
\hline 53.4 & 53 & 52.4 & 51.2 & 51.2 & 51.3 & 50.6 & 50.5 & 50 & 49.4 & 49.9 & 49.4 & \\
\hline 53.518 & 52.963 & 52.378 & 51.769 & 51.294 & 50.841 & 50.473 & 50.121 & 49.834 & 49.596 & 49.427 & 49.387 & 0.995 \\
\hline 55 & 54.6 & 53.9 & 53.4 & 52.8 & 52.7 & 52.2 & 51.8 & 51.5 & 51.4 & 51.4 & 51.4 & \\
\hline 56.86 & 56.252 & 55.616 & 54.956 & 54.431 & 53.93 & 53.514 & 53.114 & 52.78 & 52.496 & 52.282 & 52.197 & 0.954 \\
\hline 56.8 & 56.1 & 55.7 & 54.3 & 54 & 51.5 & 51.4 & 51.4 & 52.5 & 52.4 & 52.2 & 50.4 & \\
\hline 58 & 57.375 & 56.721 & 56.044 & 55.502 & 54.984 & 54.552 & 54.136 & 53.786 & 53.486 & 53.257 & 53.157 & 0.933 \\
\hline 53 & 52.8 & 52.1 & 50.8 & 49.8 & 49.4 & 49.2 & 48.4 & 47.8 & 48.1 & 48.2 & 48 & \\
\hline 53.192 & 52.642 & 52.062 & 51.458 & 50.988 & 50.54 & 50.177 & 49.829 & 49.546 & 49.313 & 49.149 & 49.113 & 0.974 \\
\hline 55.2 & 55.5 & 54.1 & 53.3 & 54.2 & 54 & 52.7 & 52.3 & 53 & 54.2 & 53.8 & 53.5 & \\
\hline 58.408 & 57.777 & 57.116 & 56.433 & 55.885 & 55.361 & 54.922 & 54.501 & 54.145 & 53.839 & 53.605 & 53.5 & 0.863 \\
\hline 57 & 56.2 & 56 & 54.8 & 54.5 & 54.2 & 53.6 & 53.8 & 53.9 & 53.1 & 53.2 & 52.4 & \\
\hline 55.963 & 55.37 & 54.747 & 54.101 & 53.589 & 53.101 & 52.698 & 52.311 & 51.989 & 51.718 & 51.516 & 51.444 & 0.944 \\
\hline 57.7 & 58 & 56.7 & 56.5 & 56 & 57.2 & 55.8 & 55.4 & 55.4 & 54.7 & 53.2 & 53.1 & \\
\hline 58.571 & 57.937 & 57.274 & 56.589 & 56.038 & 55.512 & 55.071 & 54.647 & 54.289 & 53.981 & 53.744 & 53.637 & 0.98 \\
\hline 60.3 & 60 & 58.8 & 58.1 & 57.6 & 57.4 & 56.8 & 56.1 & 55.8 & 55.3 & 55.6 & 55.7 & \\
\hline 61.749 & 61.066 & 60.354 & 59.62 & 59.022 & 58.45 & 57.963 & 57.493 & 57.091 & 56.739 & 56.46 & 56.31 & 0.958 \\
\hline 50 & 49.4 & 48.6 & 47.9 & 48.3 & 48.3 & 47.5 & 47.3 & 47.1 & 47.1 & 47.3 & 47.4 & \\
\hline 50.992 & 50.476 & 49.93 & 49.359 & 48.921 & 48.506 & 48.175 & 47.858 & 47.606 & 47.403 & 47.269 & 47.263 & 0.971 \\
\hline 63.7 & 62.8 & 62.3 & 60.7 & 60 & 59.6 & 58.8 & 58.6 & 58.1 & 57.8 & 57.2 & 57.1 & \\
\hline 66.313 & 65.558 & 64.776 & 63.974 & 63.308 & 62.668 & 62.115 & 61.581 & 61.115 & 60.7 & 60.359 & 60.148 & 0.893 \\
\hline 64.4 & 62.6 & 60.6 & 59.2 & 58.8 & 57.7 & 57.3 & 56.6 & 56.5 & 56.6 & 58.2 & 57 & \\
\hline 63.379 & 62.67 & 61.933 & 61.175 & 60.553 & 59.956 & 59.446 & 58.953 & 58.528 & 58.154 & 57.852 & 57.681 & 0.932 \\
\hline 58.8 & 58.2 & 58.2 & 57.1 & 56.5 & 56.2 & 54.6 & 55.1 & 55.2 & 57.2 & 57.9 & 55.9 & \\
\hline 60.364 & 59.702 & 59.011 & 58.299 & 57.722 & 57.169 & 56.702 & 56.253 & 55.869 & 55.537 & 55.276 & 55.145 & 0.956 \\
\hline
\end{tabular}


Table 2 Comparison of experimental data and Model result of each subject (Continued)

\begin{tabular}{|c|c|c|c|c|c|c|c|c|c|c|c|c|}
\hline 54.1 & 53.5 & 53.2 & 52.5 & 51.8 & 51.9 & 50.8 & 50.3 & 50 & 49.5 & 49.9 & 49.6 & \\
\hline 54.578 & 54.006 & 53.404 & 52.78 & 52.288 & 51.821 & 51.437 & 51.07 & 50.768 & 50.515 & 50.333 & 50.278 & 0.989 \\
\hline 67.6 & 67.8 & 67.7 & 66.2 & 65.2 & 65.1 & 64.9 & 63.8 & 62.9 & 61.8 & 61.6 & 63.2 & \\
\hline 66.476 & 65.719 & 64.934 & 64.129 & 63.461 & 62.818 & 62.263 & 61.727 & 61.258 & 60.842 & 60.498 & 60.285 & 0.896 \\
\hline 50.7 & 50.2 & 50.3 & 50.5 & 49.9 & 48.8 & 47.8 & 47.7 & 48.8 & 48.9 & 48.3 & 47.5 & \\
\hline 53.111 & 52.562 & 51.983 & 51.38 & 50.911 & 50.465 & 50.103 & 49.756 & 49.474 & 49.242 & 49.079 & 49.045 & 0.905 \\
\hline 59.6 & 59.9 & 59.6 & 58 & 57.3 & 57.6 & 57.1 & 57.5 & 57.4 & 57.2 & 56.6 & 58.1 & \\
\hline 59.875 & 59.221 & 58.537 & 57.832 & 57.262 & 56.717 & 56.257 & 55.815 & 55.438 & 55.113 & 54.858 & 54.733 & 0.917 \\
\hline 55.8 & 54.9 & 53.8 & 52.9 & 52.6 & 52.3 & 51.8 & 51.4 & 51.1 & 51.4 & 51.4 & 51.6 & \\
\hline 55.963 & 55.37 & 54.747 & 54.101 & 53.589 & 53.101 & 52.698 & 52.311 & 51.989 & 51.718 & 51.516 & 51.444 & 0.985 \\
\hline 55.7 & 55 & 54.7 & 53.5 & 52.7 & 52.6 & 51.4 & 50.8 & 51.5 & 51.4 & 51.8 & 51.8 & \\
\hline 56.452 & 55.851 & 55.221 & 54.568 & 54.048 & 53.553 & 53.143 & 52.749 & 52.42 & 52.142 & 51.934 & 51.855 & 0.974 \\
\hline 53.1 & 53.3 & 53.1 & 51.9 & 51.6 & 51.8 & 51.7 & 51.4 & 49.3 & 48.4 & 48.4 & 49.7 & \\
\hline 53.029 & 52.482 & 51.904 & 51.303 & 50.835 & 50.389 & 50.029 & 49.683 & 49.403 & 49.171 & 49.01 & 48.976 & 0.962 \\
\hline 55.5 & 54.5 & 55.2 & 53.7 & 53.4 & 53.6 & 53 & 52.8 & 53 & 51.9 & 51.8 & 51.9 & \\
\hline 55.8 & 55.209 & 54.589 & 53.946 & 53.436 & 52.951 & 52.55 & 52.165 & 51.846 & 51.576 & 51.377 & 51.306 & 0.987 \\
\hline 51.8 & 51.3 & 50.9 & 50.4 & 50.1 & 50.1 & 49.8 & 49.5 & 49.4 & 49.1 & 49 & 49.1 & \\
\hline 51.807 & 51.278 & 50.719 & 50.137 & 49.687 & 49.26 & 48.916 & 48.588 & 48.325 & 48.11 & 47.965 & 47.948 & 0.976 \\
\hline 50.4 & 50.7 & 50.2 & 48.9 & 49 & 50.3 & 50.6 & 50.9 & 50.9 & 50.4 & 49 & 49 & \\
\hline 50.747 & 50.236 & 49.693 & 49.126 & 48.692 & 48.28 & 47.952 & 47.639 & 47.391 & 47.191 & 47.06 & 47.057 & 0.776 \\
\hline 56.5 & 55.8 & 55.7 & 55.5 & 55 & 55.1 & 54.4 & 54.6 & 55.1 & 56.6 & 57.1 & 54.1 & \\
\hline 54.985 & 54.407 & 53.799 & 53.168 & 52.671 & 52.197 & 51.808 & 51.435 & 51.127 & 50.869 & 50.681 & 50.621 & 0.324 \\
\hline 64.8 & 64.5 & 63.7 & 62.2 & 61.4 & 61.5 & 60.8 & 60.2 & 59.6 & 58.9 & 59.2 & 59.5 & \\
\hline 65.091 & 64.355 & 63.592 & 62.808 & 62.16 & 61.538 & 61.003 & 60.486 & 60.037 & 59.639 & 59.314 & 59.12 & 0.996 \\
\hline
\end{tabular}

Note: 1) No. is subject number, $122(\mathrm{p})$ is actual experimental body weight value of subject 122, 122(m) is model result of subject 122 , etc. 2) $R^{2}$ is determination coefficient.

3)The experimental data of Subject 130 is not fulfilled. It shows this subject is a special case. 


\title{
Appendix B Table 3
}

Table 3 Confidence interval of estimation (Confidence level is $95 \%$ )

\begin{tabular}{|c|c|c|c|c|c|c|c|c|c|c|c|c|}
\hline Week & Weight(kg) & Weight(kg) & Weight(kg) & Weight(kg) & Weight(kg) & Weight(kg) & Weight(kg) & Weight(kg) & Weight(kg) & Weight(kg) & Weight(kg) & Weight(kg) \\
\hline & $S 13$ & S14 & S15 & S16 & S17 & S18 & S19 & S20 & S21 & S22 & S23 & S24 \\
\hline \multicolumn{13}{|l|}{ Subject No. } \\
\hline 122(p) & 51.7 & 51.5 & 51 & 50 & 49.4 & 49 & 48.5 & 48.2 & 47.7 & 47.8 & 47.2 & 47.4 \\
\hline 122(model) & $51.588 \pm 2.592$ & $51.063 \pm 2.443$ & $50.504 \pm 2.332$ & $49.919 \pm 2.241$ & $49.476 \pm 2.145$ & $49.057 \pm 2.059$ & $48.728 \pm 1.983$ & $48.414 \pm 1.919$ & $48.17 \pm 1.86$ & $47.977 \pm 1.817$ & $47.858 \pm 1.767$ & $47.876 \pm 1.745$ \\
\hline 123(p) & 55.2 & 54.9 & 54.8 & 53.9 & 53.4 & 53 & 52 & 51.9 & 51.5 & 52.1 & 52.2 & 52.1 \\
\hline 123(model) & $55.229 \pm 4.239$ & $54.645 \pm 3.992$ & $54.029 \pm 3.788$ & $53.387 \pm 3.637$ & $52.889 \pm 3.493$ & $52.415 \pm 3.364$ & $52.032 \pm 3.255$ & $51.666 \pm 3.143$ & $51.369 \pm 3.044$ & $51.125 \pm 2.952$ & $50.956 \pm 2.903$ & $50.924 \pm 2.881$ \\
\hline $119(p)$ & 53.9 & 53.4 & 53.2 & 52.2 & 51.4 & 51 & 50.8 & 50.5 & 50.3 & 50.7 & 49.8 & 49.1 \\
\hline
\end{tabular}

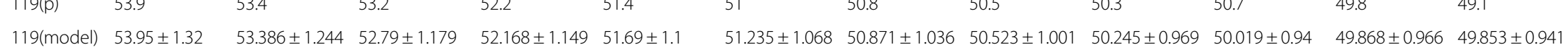
$120(p)$
$56 \quad 55.5 \quad 54.7$
54
$53.4 \quad 53$
52.3
52.1
51.3
51.2
51.6

120 (model) $\quad 57.099 \pm 1.013 \quad 56.485 \pm 0.975 \quad 55.839 \pm 0.973 \quad 55.168 \pm 0.949 \quad 54.641 \pm 0.947 \quad 54.14 \pm 0.975 \quad 53.729 \pm 1.017 \quad 53.335 \pm 1.051 \quad 53.012 \pm 1.139 \quad 52.741 \pm 1.188 \quad 52.547 \pm 1.335 \quad 52.489 \pm 1.436$
129(p) $\quad 55.8$
$55.5 \quad 54.8$
53.8
$53.4 \quad 53.4 \quad 53.3$
53.2
53
52.8
52.8
52.2

\begin{abstract}
$\begin{array}{llllllll}130(p) & 56.9 & 56.6 & 56.6 & 55.4 & 55.7 & 55.7\end{array}$
\end{abstract}
130 (model) $\quad 56.902 \pm 4.128 \quad 56.291 \pm 3.888 \quad 55.648 \pm 3.691 \quad 54.981 \pm 3.561 \quad 54.457 \pm 3.416 \quad 53.958 \pm 3.349 \quad 53.55 \pm 3.356$

$\begin{array}{lllll}55.6 & 54.2 & 53.9 & 54.5 & 53.6\end{array}$

$126(\mathrm{p})$

68.1

$67.4 \quad 67.6$

\begin{abstract}
66
\end{abstract}

\begin{abstract}
65.7
\end{abstract}

\begin{abstract}
65.4
\end{abstract}
65.3

$53.16 \pm 3.422$

$52.839 \pm 3.525 \quad 52.571 \pm 3.48$

$52.379 \pm 3.437 \quad 52.325+3.48$

$\begin{array}{lllllll}127(p) & 51.8 & 51.3 & 51.2 & 50.8 & 50.4 & 49.6\end{array}$

$49.2-48.7$

$63.353 \pm 1.691 \quad 62.869$

$62.6 \quad 61.6 \quad 60.6$

127 (model) $\quad 51.982 \pm 1.518 \quad 51.45 \pm 1.435 \quad 50.885 \pm 1.364 \quad 50.294 \pm 1.312 \quad 49.845 \pm 1.289 \quad 49.42 \pm 1.274$

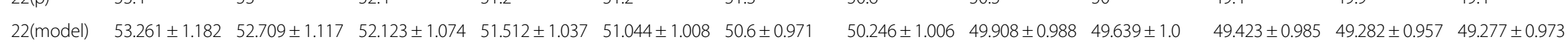
$\begin{array}{lllllllllll}23(\mathrm{p}) & 55 & 54.6 & 53.9 & 53.4 & 52.8 & 52.7 & 52.2 & 51.8 & 51.5 & 51.4\end{array}$

23 (model) $\quad 55.229 \pm 1.538 \quad 54.645 \pm 1.457 \quad 54.029 \pm 1.381 \quad 53.387 \pm 1.318 \quad 52.889 \pm 1.261 \quad 52.415 \pm 1.211 \quad 52.032 \pm 1.176 \quad 51.666 \pm 1.139 \quad 51.369 \pm 1.104 \quad 51.125 \pm 1.073 \quad 50.956 \pm 1.05 \quad 50.924 \pm 1.041$

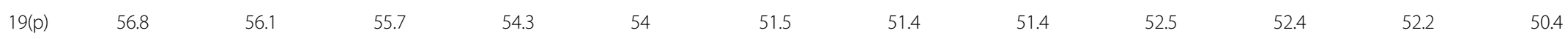

19 (model) $\quad 56.902 \pm 1.893 \quad 56.291 \pm 1.784 \quad 55.648 \pm 1.696 \quad 54.981 \pm 1.615 \quad 54.457 \pm 1.594 \quad 53.958 \pm 1.551 \quad 53.55 \pm 1.982 \quad 53.16 \pm 2.207 \quad 52.839 \pm 2.307 \quad 52.571 \pm 2.243 \quad 52.379 \pm 2.18 \quad 52.325 \pm 2.123$ $\begin{array}{llllllllllll}20(p) & 53 & 52.8 & 52.1 & 50.8 & 49.8 & 49.4 & 49.2 & 48.4 & 47.8 & 48.1\end{array}$

20 (model) $\quad 53.36 \pm 1.413 \quad 52.805 \pm 1.353 \quad 52.218 \pm 1.282 \quad 51.606 \pm 1.223 \quad 51.136 \pm 1.258 \quad 50.691 \pm 1.414 \quad 50.335 \pm 1.524 \quad 49.996 \pm 1.582 \quad 49.726 \pm 1.723 \quad 49.508 \pm 1.909 \quad 49.365 \pm 1.968 \quad 49.359 \pm 1.986$ $\begin{array}{lllllllllll}29(p) & 55.2 & 55.5 & 54.1 & 53.3 & 54.2 & 54 & 52.7 & 52.3 & 53 & 54.2\end{array}$

29 (model) $\quad 53.95 \pm 5.381 \quad 53.386 \pm 5.136 \quad 52.79 \pm 5.047 \quad 52.168 \pm 4.87 \quad 51.69 \pm 4.705 \quad 51.235 \pm 4.722 \quad 50.871 \pm 4.779 \quad 50.523 \pm 4.709 \quad 50.245 \pm 4.641 \quad 50.019 \pm 4.69 \quad 49.868 \pm 4.956 \quad 49.853 \pm 5.142$ 
Table 3 Confidence interval of estimation (Confidence level is 95\%) (Continued)

\begin{tabular}{|c|c|c|c|c|c|c|c|c|c|c|c|c|}
\hline $30(p)$ & 57 & 56.2 & 56 & 54.8 & 54.5 & 54.2 & 53.6 & 53.8 & 53.9 & 53.1 & 53.2 & 52.4 \\
\hline 30(model) & $57 \pm 2.118$ & $56.388 \pm 1.995$ & $55.743 \pm 1.895$ & $55.074 \pm 1.811$ & $54.549 \pm 1.74$ & $54.049 \pm 1.67$ & $53.639 \pm 1.611$ & $53.247 \pm 1.556$ & $52.925 \pm 1.53$ & $52.656 \pm 1.556$ & $52.463 \pm 1.525$ & $52.407 \pm 1.522$ \\
\hline $26(p)$ & 57.7 & 58 & 56.7 & 56.5 & 56 & 57.2 & 55.8 & 55.4 & 55.4 & 54.7 & 53.2 & 53.1 \\
\hline 26(model) & $57.492 \pm 1.308$ & $56.872 \pm 1.24$ & $56.22 \pm 1.373$ & $55.543 \pm 1.339$ & $55.01 \pm 1.394$ & $54.503 \pm 1.444$ & $54.086 \pm 1.995$ & $53.687 \pm 2.116$ & $53.358 \pm 2.217$ & $53.082 \pm 2.362$ & $52.881 \pm 2.416$ & $52.819 \pm 2.355$ \\
\hline $27(p)$ & 60.3 & 60 & 58.8 & 58.1 & 57.6 & 57.4 & 56.8 & 56.1 & 55.8 & 55.3 & 55.6 & 55.7 \\
\hline 27(model) & $60.149 \pm 2.482$ & $59.486 \pm 2.34$ & $58.792 \pm 2.241$ & $58.074 \pm 2.135$ & $57.501 \pm 2.043$ & $56.953 \pm 1.961$ & $56.497 \pm 1.904$ & $56.059 \pm 1.845$ & $55.692 \pm 1.786$ & $55.379 \pm 1.732$ & $55.142 \pm 1.683$ & $55.043 \pm 1.651$ \\
\hline $4(p)$ & 50 & 49.4 & 48.6 & 47.9 & 48.3 & 48.3 & 47.5 & 47.3 & 47.1 & 47.1 & 47.3 & 47.4 \\
\hline 4 (model) & $49.916 \pm 1.246$ & $49.417 \pm 1.175$ & $48.884 \pm 1.114$ & $48.325 \pm 1.074$ & $47.908 \pm 1.056$ & $47.514 \pm 1.037$ & $47.209 \pm 1.082$ & $46.92 \pm 1.055$ & $46.7 \pm 1.038$ & $46.531 \pm 1.025$ & $46.435 \pm 1.03$ & $46.476 \pm 1.077$ \\
\hline $5(p)$ & 63.7 & 62.8 & 62.3 & 60.7 & 60 & 59.6 & 58.8 & 58.6 & 58.1 & 57.8 & 57.2 & 57.1 \\
\hline 5 (model) & $63.888 \pm 2.476$ & $63.165 \pm 2.336$ & $62.412 \pm 2.226$ & $61.636 \pm 2.121$ & $61.006 \pm 2.099$ & $60.402 \pm 2.09$ & $59.891 \pm 2.057$ & $59.399 \pm 2.063$ & $58.978 \pm 2.036$ & $58.612 \pm 2.018$ & $58.323 \pm 1.997$ & $58.173 \pm 2.009$ \\
\hline $1(p)$ & 64.4 & 62.6 & 60.6 & 59.2 & 58.8 & 57.7 & 57.3 & 56.6 & 56.5 & 56.6 & 58.2 & 57 \\
\hline 1 (model) & $64.183 \pm 2.634$ & $63.456 \pm 2.485$ & $62.698 \pm 2.416$ & $61.917 \pm 2.622$ & $61.282 \pm 2.953$ & $60.674 \pm 3.146$ & $60.159 \pm 3.416$ & $59.662 \pm 3.608$ & $59.237 \pm 3.807$ & $58.867 \pm 3.918$ & $58.574 \pm 3.95$ & $58.42 \pm 3.848$ \\
\hline $2(p)$ & 58.8 & 58.2 & 58.2 & 57.1 & 56.5 & 56.2 & 54.6 & 55.1 & 55.2 & 57.2 & 57.9 & 55.9 \\
\hline 2(model) & $59.46 \pm 0.854$ & $58.808 \pm 0.916$ & $58.125 \pm 0.948$ & $57.418 \pm 0.904$ & $56.855 \pm 0.884$ & $56.318 \pm 0.871$ & $55.872 \pm 0.841$ & $55.444 \pm 1.041$ & $55.087 \pm 1.022$ & $54.783 \pm 0.992$ & $54.556 \pm 1.483$ & $54.466 \pm 2.094$ \\
\hline $11(p)$ & 54.1 & 53.5 & 53.2 & 52.5 & 51.8 & 51.9 & 50.8 & 50.3 & 50 & 49.5 & 49.9 & 49.6 \\
\hline 11 (model) & $53.852 \pm 0.897$ & $53.29 \pm 0.861$ & $52.695 \pm 0.827$ & $52.075 \pm 0.844$ & $51.597 \pm 0.843$ & $51.144 \pm 0.817$ & $50.782 \pm 0.883$ & $50.435 \pm 0.853$ & $50.158 \pm 0.828$ & $49.934 \pm 0.806$ & $49.784 \pm 0.809$ & $49.771 \pm 0.789$ \\
\hline $12(p)$ & 67.6 & 67.8 & 67.7 & 66.2 & 65.2 & 65.1 & 64.9 & 63.8 & 62.9 & 61.8 & 61.6 & 63.2 \\
\hline 12(model) & $67.922 \pm 1.654$ & $67.135 \pm 1.573$ & $66.318 \pm 1.548$ & $65.48 \pm 1.691$ & $64.787 \pm 1.67$ & $64.123 \pm 1.619$ & $63.552 \pm 1.643$ & $63.001 \pm 1.73$ & $62.523 \pm 1.72$ & $62.1 \pm 1.678$ & $61.755 \pm 1.636$ & $61.551 \pm 1.593$ \\
\hline $8(p)$ & 50.7 & 50.2 & 50.3 & 50.5 & 49.9 & 48.8 & 47.8 & 47.7 & 48.8 & 48.9 & 48.3 & 47.5 \\
\hline 8 (model) & $53.36 \pm 4.876$ & $52.805 \pm 4.92$ & $52.218 \pm 4.943$ & $51.606 \pm 4.846$ & $51.136 \pm 4.68$ & $50.691 \pm 4.543$ & $50.335 \pm 4.49$ & $49.996 \pm 4.525$ & $49.726 \pm 4.524$ & $49.508 \pm 4.409$ & $49.365 \pm 4.292$ & $49.359 \pm 4.205$ \\
\hline $9(p)$ & 59.6 & 59.9 & 59.6 & 58 & 57.3 & 57.6 & 57.1 & 57.5 & 57.4 & 57.2 & 56.6 & 58.1 \\
\hline 9 (model) & $59.755 \pm 3.082$ & $59.099 \pm 2.905$ & $58.411 \pm 2.799$ & $57.699 \pm 2.759$ & $57.132 \pm 2.646$ & $56.59 \pm 2.541$ & $56.14 \pm 2.506$ & $55.708 \pm 2.469$ & $55.346 \pm 2.548$ & $55.039 \pm 2.661$ & $54.807 \pm 2.774$ & $54.714 \pm 2.82$ \\
\hline 104(p) & 55.8 & 54.9 & 53.8 & 52.9 & 52.6 & 52.3 & 51.8 & 51.4 & 51.1 & 51.4 & 51.4 & 51.6 \\
\hline 104(model) & $56.016 \pm 1.381$ & $55.42 \pm 1.308$ & $54.791 \pm 1.282$ & $54.137 \pm 1.358$ & $53.627 \pm 1.48$ & $53.141 \pm 1.529$ & $52.746 \pm 1.539$ & $52.369 \pm 1.563$ & $52.061 \pm 1.587$ & $51.806 \pm 1.606$ & $51.626 \pm 1.572$ & $51.583 \pm 1.533$ \\
\hline 105(p) & 55.7 & 55 & 54.7 & 53.5 & 52.7 & 52.6 & 51.4 & 50.8 & 51.5 & 51.4 & 51.8 & 51.8 \\
\hline 105(model) & $55.918 \pm 1.544$ & $55.323 \pm 1.462$ & $54.695 \pm 1.401$ & $54.043 \pm 1.334$ & $53.534 \pm 1.314$ & $53.05 \pm 1.342$ & $52.657 \pm 1.314$ & $52.281 \pm 1.423$ & $51.974 \pm 1.56$ & $51.721 \pm 1.53$ & $51.542 \pm 1.493$ & $51.501 \pm 1.458$ \\
\hline $101(p)$ & 53.1 & 53.3 & 53.1 & 51.9 & 51.6 & 51.8 & 51.7 & 51.4 & 49.3 & 48.4 & 48.4 & 49.7 \\
\hline 101 (model) & $53.064 \pm 2.0$ & $52.515 \pm 1.884$ & $51.933 \pm 1.853$ & $51.325 \pm 1.898$ & $50.859 \pm 1.846$ & $50.418 \pm 1.818$ & $50.067 \pm 1.898$ & $49.732 \pm 2.015$ & $49.467 \pm 2.117$ & $49.253 \pm 2.054$ & $49.114 \pm 2.035$ & $49.112 \pm 2.007$ \\
\hline 102(p) & 55.5 & 54.5 & 55.2 & 53.7 & 53.4 & 53.6 & 53 & 52.8 & 53 & 51.9 & 51.8 & 51.9 \\
\hline 102(model) & $55.721 \pm 1.479$ & $55.129 \pm 1.401$ & $54.505 \pm 1.385$ & $53.856 \pm 1.383$ & $53.35 \pm 1.327$ & $52.869 \pm 1.274$ & $52.478 \pm 1.287$ & $52.105 \pm 1.271$ & $51.801 \pm 1.277$ & $51.55 \pm 1.365$ & $51.374 \pm 1.336$ & $51.336 \pm 1.315$ \\
\hline $111(p)$ & 51.8 & 51.3 & 50.9 & 50.4 & 50.1 & 50.1 & 49.8 & 49.5 & 49.4 & 49.1 & 49 & 49.1 \\
\hline 111 (model) & $51.588 \pm 0.896$ & $51.063 \pm 0.855$ & $50.504 \pm 0.824$ & $49.919 \pm 0.82$ & $49.476 \pm 0.832$ & $49.057 \pm 0.869$ & $48.728 \pm 1.003$ & $48.414 \pm 1.113$ & $48.17 \pm 1.204$ & $47.977 \pm 1.308$ & $47.858 \pm 1.375$ & $47.876 \pm 1.435$ \\
\hline
\end{tabular}


Table 3 Confidence interval of estimation (Confidence level is $\mathbf{9 5 \% )}$ (Continued)

\begin{tabular}{|c|c|c|c|c|c|c|c|c|c|c|c|c|}
\hline $112(p)$ & 50.4 & 50.7 & 50.2 & 48.9 & 49 & 50.3 & 50.6 & 50.9 & 50.9 & 50.4 & 49 & 49 \\
\hline 112(model) & $50.014 \pm 1.162$ & $49.514 \pm 1.124$ & $48.979 \pm 1.3$ & $48.419 \pm 1.438$ & $48 \pm 1.404$ & $47.605 \pm 1.455$ & $47.299 \pm 2.001$ & $47.008 \pm 2.567$ & $46.786 \pm 3.144$ & $46.616 \pm 3.632$ & $46.519 \pm 3.945$ & $46.558 \pm 4.001$ \\
\hline 108(p) & 56.5 & 55.8 & 55.7 & 55.5 & 55 & 55.1 & 54.4 & 54.6 & 55.1 & 56.6 & 57.1 & 54.1 \\
\hline 108(model) & $56.804 \pm 4.93$ & $56.194 \pm 4.648$ & $55.553 \pm 4.413$ & $54.887 \pm 4.204$ & $54.364 \pm 4.038$ & $53.867 \pm 3.891$ & $53.461 \pm 3.805$ & $53.072 \pm 3.705$ & $52.752 \pm 3.664$ & $52.486 \pm 3.728$ & $52.295 \pm 4.098$ & $52.242 \pm 4.545$ \\
\hline 109(p) & 64.8 & 64.5 & 63.7 & 62.2 & 61.4 & 61.5 & 60.8 & 60.2 & 59.6 & 58.9 & 59.2 & 59.5 \\
\hline 109(model) & $64.675 \pm 0.839$ & $63.94 \pm 0.795$ & $63.174 \pm 0.832$ & $62.386 \pm 0.852$ & $61.744 \pm 0.823$ & $61.128 \pm 0.812$ & $60.605 \pm 0.807$ & $60.102 \pm 0.785$ & $59.669 \pm 0.761$ & $59.293 \pm 0.739$ & $58.993 \pm 0.741$ & $58.832 \pm 0.727$ \\
\hline
\end{tabular}

2) In all 384 confidence intervals, 26 actual body weight values are outside the confidence interval, but 9 values from these 26 values are within the area of statistical handling error. So the unsatisfied rate of estimation is

from $4.4 \%$ to $6.77 \%$. It shows our model estimation is acceptable. 3)a is $5 \%$. The confidence interval: estimated body weight $\pm t_{\alpha / 2}(n-2) * S E$, where $S E$ is standard error, degree of freedom is $n-2$. 


\section{Competing interests}

The authors declare that they have no competing interests.

\section{Authors' contributions}

ZG (Zhejun Gong) conceived the idea and wrote the manuscript. ZG (Zhefeng Gong) collected the experimental data. All authors read and approved the final manuscript.

\section{Reviewer's report}

\section{Reviewer 1 (Dr. E. Cabral Balreira)}

The authors propose an interesting model between Energy intake and body weight based on Fick's second law of diffusion. This is an important are of research and the present article provides an interesting contribution to this research area and can provide new insight into the body weight mechanisms. The work justifying the use of Fick's second law of diffusion went to a substantial justification and it is well motivated and explained. The referee agrees with the authors that there should be an investigation of the model. The referee recommends that a major revision of the paper is required in order to be published in the Biology Direct.

The main issues are outlined as follows.

- In the results section, the authors did not fully disclosed their hypotheses that the energy density of body mass is independent of time. They needed this fact to arrive at their model equation (4). This is not supported by the previous discussion and it is big hypotheses that needs more explanation.

Author reply: Generally, for adult men (20-33y) in the Minnesota human starvation study, the change in body weight is largely due to fat mass (FM), but not fat-free mass(FFM). As we can see from Kyle et al., fat-free mass does not change much at middle age (from 18-34y to 35-59y), especially when compared with fat mass which changes significantly during the same period [17]. Considering that the energy density of FM is much higher than that of FFM, the energy change is largely decided by change in FM. Thus, the change in energy intake, $d\left(\rho^{*} V\right)$, is approximately the change in energy in fat mass, which can be represented as $p^{*} d(V)$, in which $p$ is the energy density of fat mass. The energy density of fat mass is supposed to be a constant, so we think the formula $d\left(\rho^{*} V\right)=p^{*} d V$ is valid and the possible error here won't affect our conclusion significantly.

- In the section titled simulation of body weight change using the developed model, it is unclear how the authors obtained the experimental data.

Author reply: Total Energy Expenditure (TEE) includes two major parts: Resting Energy Expenditure (REE), the amount of calories needed to maintain basic body systems and body temperature at rest; Activity Energy Expenditure (AEE), the amount of calories used during activity [20]. Net energy intake is the difference between food intake and TEE. Although TEE was not measured in the Minnesota starvation study, TEE can be obtained through calculating REE and AEE $[19,21]$.

REE is calculated from Basal oxygen (cc/min) and kcalorie equivalent per ccl min. The daily energy expenditure at rest converts cc of oxygen/min into liters of oxygen/day, multiplied by the kcalorie equivalent of oxygen. The caloric equivalent of each cc of oxygen consumed in the resting state is calculated on the basis of Thorne Martin Carpenter's 1921 table [19]. The group's REE of $994.2 \mathrm{kcal} /$ day at $\mathrm{S} 24$ equals group oxygen consumption of $139.1 \mathrm{cc} / \mathrm{min}$ multiplied by 1.44 (1440 min divided by 1000) and the groups' caloric equivalent of oxygen of $4.964 \mathrm{kcal} / \mathrm{cc}$.

We here give an example to show how AEE is calculated. 22 miles per week of outdoor walking means 3.14 miles walking per day. A man's normal walking speed is 3 miles per hour or so. When a $54 \mathrm{~kg}$ man walks with speed of $3 \mathrm{mph}$, the energy expenditure is $3.6 \mathrm{kcal} / \mathrm{min}$. At S24, the group's body weight is $52.57 \mathrm{~kg}$. The group's energy expenditure is $(52.57 / 54) *(3.14 / 3) * 3.6 * 60=220.1$ (kcal/day) [21]. When a $54 \mathrm{~kg}$ subject walks at $3.5 \mathrm{mph}$ for half hour per week on a treadmill, his energy expenditure is $4.2 \mathrm{kcal} / \mathrm{min}$. The group's energy expenditure is $(52.57 / 54) * 4.2 * 30 / 7=17.52$ (kcal/day) [21]. These two parts of walking energy expenditure added, we can know AEE is $237.62 \mathrm{kcal} /$ day. So at S24, TEE is $1231.83 \mathrm{kcal} /$ day, net energy intake is $409.8 \mathrm{kcal} /$ day.
From their work, in page 8 below equation (9), the authors state that they simply generated data from their own model and use that same data to validate the model. Such approach is circular and does not support the model validation. It simply shows that the ISCEM algorithm is working properly.

The authors must validate their model using the actual experimental data which they display in Table 1. Using the data from the Minnesota human starvation study, the authors need to estimate the parameters of their model, plot the actual results against the model predictions and report the $\mathrm{R}^{2}$ value.

Author reply: In fact, we actually estimated the model parameters using the experimental data from Table 1. We actually used the experimental data from Table 1 to validate the model. We also plotted the actual experimental results against the model predictions and reported the $R^{2}$ value.

- Finally, the authors need to better explain how the ISCEM algorithm works and how is the SCEM-UA algorithm optimizing the parameters in their nonlinear problem.

Author reply: Corrected.

\section{Reviewer 2 (Prof. Yang Kuang)}

This paper address an interesting but potentially controversial modeling problem that due to the quality or simplicity of the data, may be modeled by other simple or simpler models. There seems to be no real difficulties in fitting the data sets used in the three Figures. For example, using the first few weeks' data, we can find a energy and mass conversion rate for each subject and then use their weekly Total Energy Expenditure (TEE) to predict their weekly weight. Maybe the authors can comment on why such a simple and intuitive approach was not explored?

Author reply: We proposed a molecular diffusion based model to uncover the relationship between energy intake and body weight. We used the data from the Minnesota human starvation study to verify the validity of our molecular diffusion based model. Because the relationship between body weight and energy intake is not linear, to predict body weight simply using the energy and mass conversion rate is not feasible, even if from a pure data fitting purpose.

\section{Reviewer 3 (Dr. Chao Chen)}

The authors propose a mathematical model in which body weight at time $t$ is a function of linear combination of an error function, erf(\#/\#t) (a monotonic increasing function), and its complement 1-erf(\#/\#t)(a monotonic decreasing function), derived from the hypothesis of molecular diffusion following Fick's second law. The model is found to have a good fit to a set of data taken from the Minnesota human starvation study. However, only data from the second phase of the study during the 24 weeks starvation period are used for model fitting; excluding data of the control and recovery phases from the same study.

Author reply: In order to make clear how the body weight is affected by energy intake, we chose the data of starvation period from the Minnesota human starvation study.

The authors claim: "This model provides valuable insights into the neural basis of behavioral decisions and their resulting effects". It is difficult to see, on the basis of the presentation, any mechanistic connection as claimed. This article is just a data fitting exercise because similar models that are linear combination of two monotonic functions of opposing trends can also adequately fit the data.

Author reply: This sentence, "This model provides valuable insights into the neural basis of behavioral decisions and their resulting effects", is deleted.

We considered that molecular diffusion (of, for example, neuropeptides) plays an important role in body weight changes. Because molecular diffusion is 
accompanied by energy transference, we then describe the molecular diffusion based process with energy diffusion.

Our purpose is not to do data fitting exercise, but to use the data from the Minnesota human starvation study to verify the validity of our molecular diffusion based model.

Furthermore, this data fitting exercise leaves a lot to be desired: e.g., only the mean body weight over time were analyzed, as presented in Figures 1-3; no body weight changes from individual's baseline was analyzed; and no statistical analysis, such as confidence intervals, for predicted body weight changes were provided.

Author reply: Please see Appendix A and Appendix B.

Editorial issues:

Pages 7-8. Something must be wrong: it is unlikely that parameters are estimated to be identical when different data sets from S1-S24 and S1-S12 are used.

\section{Author reply: Corrected.}

First line on top of p9: "are" should be deleted.

Author reply: Corrected.

\section{Acknowledgment}

This research is supported by the National Natural Science Foundation of China (No. 31070944).

Received: 22 March 2012 Accepted: 13 June 2012

Published: 29 June 2012

\section{References}

1. Richards MP, Proszkowiec-Weglarz M: Mechanisms regulating feed intake, energy expenditure, and body weight in poultry. Poult Sci 2007, 86:1478-1490.

2. Vogels N, Westerterp-Plantenga MS: Categorical strategies based on subject characteristics of dietary restraint and physical activity, for weight maintenance. Int J Obes 2005, 29:849-857.

3. Hill JO: Understanding and addressing the epidemic of obesity: an energy balance perspective. Endocr Rev 2006, 27:750-761.

4. Rowland NE, Vaughan $\mathrm{CH}$, Mathes $\mathrm{CM}$, Mitra A: Feeding behavior, obesity, and neuroeconomics. Physiol Behav 2008, 93:97-109.

5. Guo J, Hall KD: Estimating the continuous-time dynamics of energy and fat metabolism in mice. PLoS Comput Biol 2009, 5:e1000511.

6. Guo J, Hall KD: Predicting changes of body weight, body fat, energy expenditure and metabolic fuel selection in C57BL/6 mice. PLoS One 2011, 6:e15961.

7. Chow CC, Hall KD: The dynamics of human body weight change. PLoS Comput Biol 2008, 4:e1000045.

8. Guo J, Jou W, Gavrilova O, Hall KD: Persistent diet-induced obesity in male C57BL/6 mice resulting from temporary obesigenic diets. PLoS One 2009, 4:e5370.

9. Takahashi T: Toward molecular neuroeconomics of obesity. Med Hypotheses 2010, 75:393-396.

10. Gortari PD, Joseph-Bravo P: Neuroendocrine regulation of energy homeostasis. Molecular Endocrinology 2006, 65:65-85.

11. Grill HJ: Distributed neural control of energy balance: contributions from hindbrain and hypothalamus. Obesity (Silver Spring) 2006, 5(Suppl):216S-221S.

12. Shioda S, Takenoya F, Yagi M, Wang L, Hori Y, Kageyama H: Neural networks of several novel neuropeptides involved in feeding regulation. Nutrition 2008, 24:848-853.

13. Siegel GJ, Agranoff BW, Albers RW, Fisher SK, Uhler MD: Basic Neurochemistry: Molecular, Cellular and Medical Aspects. 6th edition. Philadelphia: Lippincott-Raven; 1999.

14. Ludwig M, Leng G: Dendritic peptide release and peptide-dependent behaviours. Nat Rev Neurosci 2006, 7:126-136.

15. Nässel DR: Neuropeptide signaling near and far: how localized and timed is the action of neuropeptides in brain circuits? Invert Neurosci 2009, 9:57-75.
16. Smith WF: Foundations of Materials Science and Engineering,3rd edition. New York: McGraw-Hill; 2004.

17. Kyle UG, Genton L, Hans D, Karsegard L, Slosman DO, Pichard C: Agerelated differences in fat-free mass, skeletal muscle, body cell mass and fat mass between 18 and 94 years. European Journal Clinical Nutrition 2001, 55:663-672.

18. Gong Z, Gong Z: A molecular diffusion based utility model for Drosophila larval phototaxis. Theoretical Biology and Medical Modelling 2012, 9:3.

19. Hargrove $J$, Heinz G, Heinz O: Modeling transitions in body composition: the approach to steady state for anthropometric measures and physiological functions in the Minnesota human starvation study. Dynamic Medicine 2008, 7:16.

20. Ball EJ, O'Connor J, Abbott R, Steinbeck KS, Davies PSW, Wishart C, Gaskin KJ, Baur LA: Total energy expenditure, body fatness, and physical activity in children aged 6-9 y. Am J Clin Nutr 2001, 74:524-8.

21. MCARDLE WD, et al: Essentials of Exercise Physiology. 2nd edition. USA: Lippincott Williams and Wilkins; 2000.

22. Vrugt JA, Gupta HV, Bouten W, Sorooshian S: A Shuffled Complex Evolution Metropolis algorithm for optimization and uncertainty assessment of hydrological model parameters. Water Resour Res 2003, 39:1201-1216.

doi:10.1186/1745-6150-7-19

Cite this article as: Gong and Gong: Modeling the relationship between body weight and energy intake: A molecular diffusion-based approach. Biology Direct 2012 7:19.

\section{Submit your next manuscript to BioMed Central and take full advantage of:}

- Convenient online submission

- Thorough peer review

- No space constraints or color figure charges

- Immediate publication on acceptance

- Inclusion in PubMed, CAS, Scopus and Google Scholar

- Research which is freely available for redistribution

Submit your manuscript at www.biomedcentral.com/submit
C) Biomed Central 